\title{
The wandering spleen
}

\author{
C A Maxwell-Armstrong, E D V Clarke, T M Tsang, R J Stewart
}

\begin{abstract}
Wandering spleen is a rare condition that commonly presents with splenic infarction secondary to torsion. Splenectomy was previously advocated as the treatment of choice, though recent reports suggest that the organ may be detorted, and splenopexy performed. This paper documents how the latter procedure may be done using adherent omentum.

(Arch Dis Child 1996; 74: 247-248)
\end{abstract}

Keywords: greater omentum, splenopexy, wandering spleen, omental adhesions.

Wandering spleen was originally described in the 19th century, and is characterised by a lack of the normal ligamentous attachments of the organ to the diaphragm, colon, and retroperitoneum. It is a rare condition, with the majority of cases presenting as an acute abdomen, associated with torsion and subsequent splenic infarction. In most this has necessitated splenectomy, though recent reports suggest that preservation of the spleen is possible following untwisting of the hilar vessels, and subsequent splenopexy. Various techniques have been described for this latter procedure. These have included formation of an extraperitoneal pocket, suture splenopexy, and colonic displacement.

We report use of the greater omentum for elective splenopexy in a boy with intermittent splenic torsion.

\section{Case report}

An 11 year old boy was admitted as an emergency with a four day history of left upper

Nottingham NG7 2UH.

Accepted 16 November 1995

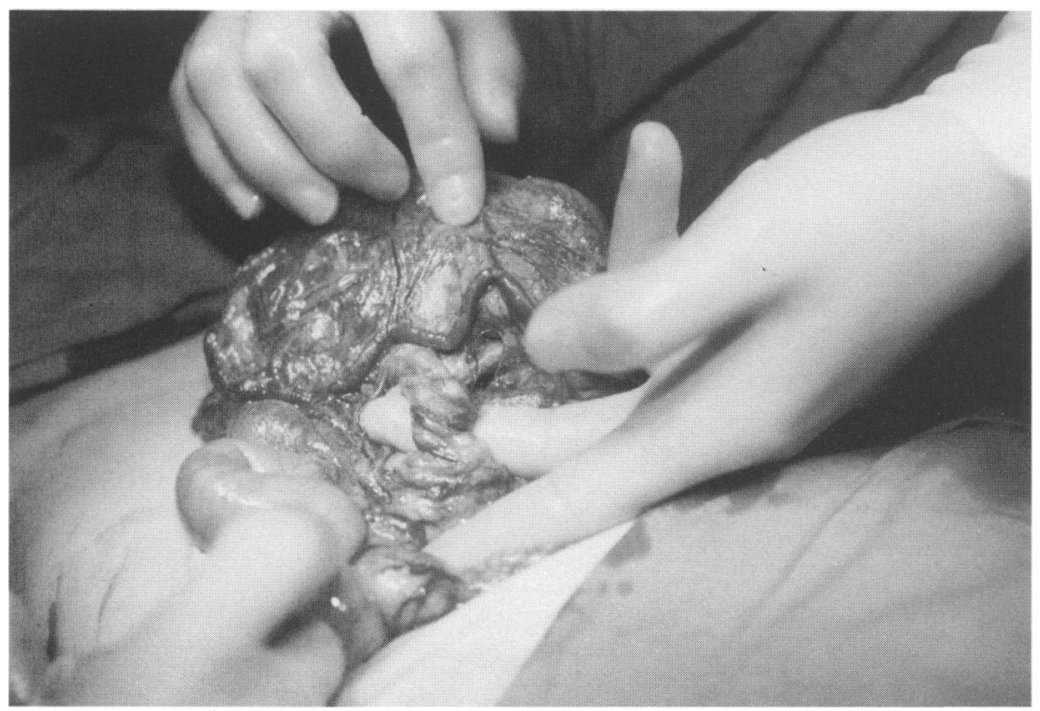

Intraoperative photograph showing torsion of the splenic pedicle.

quadrant pain. It was colicky in nature, and relieved by vomiting and curling up. He had been seen by his general practitioner on six separate occasions with similar pain over the previous seven years. Each time the pain settled spontaneously, and no diagnosis had as yet been made. He was known to have congenital mitral stenosis, and a mobile spleen, which could be manipulated to all quadrants of the abdomen. On examination he was afebrile, with a moderately tender, palpable spleen in the left hypochondrium. His symptoms rapidly resolved, and a subsequent ultrasound scan showed the spleen to be engorged, with normal echogenicity and no focal lesions. A Doppler scan confirmed normal flow in the splenic vessels. His pain recurred within one week, and on examination a tender central abdominal mass was now present, with no spleen palpable in the left upper quadrant. A liver-spleen technetium scan revealed poor splenic uptake, thus supporting the diagnosis of splenic torsion.

At laparotomy the spleen was found to be engorged, and completely wrapped in greater omentum adherent to its surface. There was a 720 degree anticlockwise torsion of the splenic pedicle (figure). The gastrosplenic ligament was absent, and there was a non-rotation of the midgut. The adherent omentum was mobilised from the splenic pedicle, and the spleen detorted. After several minutes, the spleen became less engorged, and normal colour returned. Splenopexy was performed with six interrupted 3-0 polydioxanone (PDS) sutures, anchoring the spleen by the covering omentum in the left upper quadrant. A gastropexy was performed with interrupted PDS in order to recreate the gastrosplenic ligament. His postoperative recovery was unremarkable, and he was discharged six days after the procedure. He remained asymptomatic at six weeks follow up, and a repeat liver-spleen technetium scan revealed normal uptake of isotope in his spleen.

\section{Discussion}

Wandering spleen is a rare condition thought to be due to abnormal development of the dorsal mesogastrium. ${ }^{1}$ It may present with acute or chronic abdominal pain. The former is more likely, and is due to torsion and splenic infarction. $^{2}$ Other complications include gastrointestinal obstruction secondary to splenic adhesions or a long splenic pedicle, pancreatic necrosis, bleeding from gastric varices, and abscess formation. ${ }^{3-6}$

In this case, the history was chronic, with an acute exacerbation precipitating admission. The diagnosis was suggested by the known splenic mobility, and the finding of a tender spleen. This was supported by the poor splenic 
uptake on liver-spleen isotope scan. Such a combination of findings has been shown by others to be consistent with splenic torsion, ${ }^{7}$ though computed tomography was used as opposed to ultrasound. Other papers recommend sonography as a reliable examination. ${ }^{1}$ Non-operative treatment of a wandering spleen is associated with a complication rate as high as $65 \% .^{8}$ Consequently splenectomy and splenopexy represent the only surgical treatment options. The former, though initially popular, 21112 has been discredited, as postsplenectomy sepsis may occur, with a mortality of $30-60 \% .^{9} 10$ This is particularly pertinent in our patient, who may ultimately need mitral valve replacement. With the emphasis currently on preservation, a variety of techniques has been described for splenopexy of the wandering spleen. These have included suturing the organ by its capsule to the left upper quadrant, ${ }^{113}$ and forming a posterolateral extraperitoneal pocket at the level of the 12 th rib. ${ }^{14}$ Other methods have included dislocating the left transverse colon in front of the replaced spleen, and suturing the greater curvature of the stomach to the anterior abdominal wall. ${ }^{15}$ More recently the use of a polyglycolic mesh as a 'snood', to anchor the spleen, has been reported. ${ }^{16}$ In this patient the fortuitous discovery of greater omentum adherent to the spleen enabled us to anchor the organ in the left upper quadrant. To the best of our knowledge this has not previously been described. Our technique is advantageous in that it avoids the use of prosthetic material, and is less traumatic to the spleen. We recommend this as an alternative approach for splenic fixation in the limited number of cases where greater omentum is found at laparotomy to be adherent to the spleen.

1 Stringel G, Soucy P, Mercer S. Torsion of the wandering spleen: splenectomy or splenopexy? F Pediatr Surg 1982 17: 373-5.

2 Abell I. Wandering spleen with torsion of the pedicle. Ann Surg 1933; 98: 722-35.

3 Keidar S, Freud M, Rosenschein S, et al. Intestinal obstruction due to a wandering spleen. Am $\mathcal{F}$ Gastroenterol 1978; 69: 701-4.

4 Shende A, Canzkowsky P, Becken J. Torsion of a visceroptosed spleen. Am $\mathcal{F}$ Dis Child 1976; 130: 88-91.

5 Vermylen C, Lebecque P, Claus D, et al. The wandering spleen. Eur $\mathcal{Y}$ Pediatr 1983; 140: 112-5.

6 Daneshgar S, Eras P, Feldman S, et al. Bleeding gastric varices and gastric torsion secondary to a wandering spleen. Gastroenterology 1980; 79: 141-3

7 Toback AC, Steece DM, Kaye MD. Splenic torsion. An unusual cause of splenomegaly. Dig Dis Sci 1984; 29: 868-71.

8 Thompson J, Ross R, Pizzaro S. The wandering spleen in infancy and childhood. Clin Pediatr 1980; 19: 221-4.

9 Singer DB. Postsplenectomy sepsis. In: Rosenburg HS, Bolande RP, eds. Perspective in pediatric pathology. Chicago: Year Book Medical Publishers, 1973: 285-311.

10 Balfanz JR, Nesbit ME, Jarvis C, et al. Overwhelming sepsis following splenectomy for trauma. $\mathcal{F}$ Pediatr 1976; 88: 458-9.

11 Bohrer VJ. Torsion of the wandering spleen. Ann Surg 1940; 113: 416-26.

12 Hinck VC. Wandering spleen. Norwest Med 1961; 60: $1183-5$.

13 Jones BJ, Daly M, Delaney PV. Torsion of the spleen managed by splenopexy. Br f Surg 1991; 78: 887-8.

14 Seashore JH, McIntosh S. Elective splenopexy for wandering spleen. F Pediatr Surg 1990: 25: 270-2.

15 Caracicolo F, Bonatti PC, Castruci G, et al. Wandering spleen. Treatment with colonic displacement. $f R$ Coll Surg Edinb 1986; 31: 242-4.

16 Schmidt SP, Gibbs Andrews $H$, White J. The splenic snood: an improved approach for the management of the wandering spleen. $\mathcal{F}$ Pediatr Surg 1992; 27: 1043-4. 